Methodological aspects and updates of computational models

\title{
Methodological aspects of the implementation of the new ICRP recommendations
}

\author{
W. RASKOB, C. LANDMAN
}

\begin{abstract}
With the ICRP recommendations Publications 103, 109 and 111 (ICRP, 2007; ICRP 2009a, 2009b), new concepts and quantities have been introduced into emergency management and rehabilitation. Two of them will possibly influence national procedures, but for sure they will influence countermeasure simulation approaches: 1 . The concept of a "reference level" for emergency and existing controllable exposure situations that represents the level of dose or risk, above which it is judged to be inappropriate to plan to allow exposures to occur, and for which therefore protective actions should be planned in advance. 2 . When deciding on the optimum course of protective actions, all exposure pathways and all relevant actions have to be taken into account. The major changes for the simulation models result from the second recommendation that all exposure pathways must be considered when deciding on protective actions. So far all countermeasure simulations in the early phase of an emergency are carried out by considering individual countermeasures such as sheltering or evacuation, if some dose limit for the respective action is exceeded. This approach has to be changed and strategies of several countermeasures analysed and simulated with the ultimate goal not to exceed the reference level over a given time period, typically one year.
\end{abstract}

Keywords: Countermeasure simulation/methodology/reference level/decision support/RODOS

\section{Introduction}

In 2007, the new ICRP Recommendations (ICRP, 2007) on radiation protection principles were issued presenting a distinct evolution of the former ones issued more than 15 years earlier (ICRP, 1991). These recommendations play an

KIT - Karlsruhe Institute of Technology, Hermann-von-Helmholtz-Platz 1, 76344 Eggenstein-Leopoldshafen, Germany. 
important role as they influence national, European or even international standards that will become national or international law at one point in time. The ICRP Publication 60 recommendations were based on the concept of dose saved arguing that a countermeasure should be introduced as soon as the result, the dose saved, justifies the measure. Countermeasures are treated separately resulting in intervention levels triggering the initiation of each of the various countermeasures individually.

The concept of a "reference level" is proposed for emergency and existing controllable exposure situations that represents the level of dose or risk, above which it is judged to be inappropriate to plan to allow exposures to occur, and for which therefore protective actions should be planned in advance. This concept as a consequence requires an integrated treatment of all exposure pathways for accidental and existing exposure situations thus differing considerably from the existing concept of single exposure pathways resulting in actions such as sheltering, evacuation and distribution of stable iodine tablets.

The reference level and the integrated management approach results in a new target for the preplanning and optimisation that is called "residual dose" after a certain time period, typically defined as one year. This value can be seen as the target for any management strategy. None of the existing models can deal with these new recommendations, even if some effort was spent in the RODOS system (Ehrhardt, 2000; Raskob, 2010) to have at least a demonstration of the potential effect of the ICRP recommendations on the countermeasure management.

\section{Status of modeling}

All European member states use intervention criteria for individual countermeasures. This is reflected in the mathematical models implemented in modern Decision Support Systems such as ARGOS (Hoe, 2001) and RODOS. In RODOS for example the model for early countermeasures comprises individual sub-models for sheltering, evacuation and iodine prophylaxis, only coupled in their timing parameters but separated in terms of initiation criteria. Furthermore, the late phase models for inhabited areas and food production systems are also separate entities optimised for the usage in their individual filed of modelling.

Further to this, at present, the approaches used in Europe for the definition of the intervention limit differ from country to country. The most serious difference is the usage of the projected dose or the averted dose. The latter one is in line with the "old" ICRP recommendations whereas the projected dose reflects the new recommendations. For example the 23 countries represented in the RODOS system use both the averted dose (14 countries) and the projected dose ( 9 countries).

In 2007, Heads of European Radiation Control Authorities launched an initiative to harmonise European action levels for early phase countermeasures. A working group with the name "Emergency Preparedness and Action Levels" (EPAL) was established in which 13 European countries aim to find consensus and also consider new recommendations such as the ICRP Publication 103. Results from 
this group or the Expert Group on Implementation of New International Recommendations for Emergency Exposure Situations (EGIRES) of the Working Party of Nuclear Emergency Matters (WPNEM) of the Nuclear Energy Agency (NEA) will be an important input to potential modifications of the simulation models.

\section{Methodolical challenges of the new recommendations}

As part of the NERIS Platform a working group has been set up to support the model development. The objective of that working group is to provide guidance on the methodological aspects and the operational application of the new simulation models. Within the first meeting in November 2010, this group identified a list of challenges and topics that should be considered when developing new simulation models.

Questions have been raised such as:

1. Is the "residual dose" the right target for decision making?

2. Should the new models support the use of operational intervention levels (OILs) and emergency action levels (EALs) as trigger levels for initiating a countermeasure strategy?

3. Is there a possibility to stay with the individual intervention levels but optimise them in advance that they fit to the reference level?

4. Is the distribution of iodine tablets a single measure with its own criterion or part of a strategy?

5. Is there a need to have more than one reference level, dependent on the threat?

6. How to define the dose criteria for the lifting of measures?

7. Is the dose from food part of the simulation strategy or should a dose from food consumption of $5 \mathrm{mSv}$ be assumed as maximum related to the current maximum concentration levels in food?

Consensus was reached, that OILs and EALs are important for the operational community, in particular for the fast decision making in case of an emergency. As these values do not exist at the moment, it was requested, that the new models should be applicable to derive such values based on scenario calculations.

It was also agreed that the contribution of the foodchain should be considered together with all the other exposure pathways. This implies that information about consumption habits and about critical groups can be used for the calculations.

There was no agreement so far related to the problem of lifting of early phase countermeasures and the dose that is used to derive the reference value or the residual dose. In particular how to calculate the projected residual dose is still an open question. Some organisations favour the potential dose not taking credit for the normal living, whereas others opt for the usage of the best estimate which includes normal living conditions and no critical groups for the ingestion pathways. 
To overcome this issue new simulation models might be designed in a way to consider all these various options.

A further challenge can be seen in the request to optimise countermeasures with time. Optimisation was always part of any recommendation but so far never realised in simulation models nor described in detail how to be realised in the decision making process. There are statements that optimisation should consider not only hard facts but also soft ones (ICRP, 2007), however, the operational applicability of these recommendations is questionable. Therefore, the working group requested that the new simulation models, in particular for the late phase countermeasures such as decontamination and food, should include specific optimisation routines that allow the decision maker to optimise countermeasure strategies as soon as new information is available.

Including all exposure pathways in one simulation model together with possibilities to define strategies and to optimise them with time might result in a complex user interface that is not user friendly and difficult to operate. Therefore, the design of the new model has to be performed together with their users to assure that the current usability will not be traded against functionality. Furthermore, the optimisation process requires intensive user interaction and this might again be in contrast to the simple usage when checking pre-defined countermeasure strategies.

\section{Conclusions}

The new simulation models that will support the application of the new ICRP recommendations have to be flexible and should be designed in a way to derive necessary information for the operational realisation of the new recommendations on a European or national level. It was agreed that ICRP Publication 103 provides a great opportunity to harmonise methods and regulations on a European wide level, given the fact that a simulation environment will be available that allows to define best estimate solutions.

Key challenges for the new models are:

- Include all exposure pathways in a consistent way in one simulation model.

- Provide a flexibility that allows assessing different potential threat scenarios to derive OILs and EALs.

- Provide a methodological approach and guidance to optimise late phase countermeasures such as decontamination and ingestion.

- Usability of the new models should not be lost due to a more comprehensive modelling.

To answer these challenges, a NERIS-TP working group has met and a design document has been prepared for comment by all potential end users including the NERIS Platform ICRP working group. Decisions taken so far will be presented in a second paper describing the proposed model realisation. 


\section{REFERENCES}

Ehrhardt J., Weis A. (eds) (2000), RODOS: Decision Support System for Offsite Nuclear Emergency Management in Europe. European Commission, Brussels, Report EUR 19144.

Hoe S., Müller H., Gering F., Thykier-Nielsen S., Havskov Sorensen J., Argos (2001) A decision support system for nuclear emergencies. In: American Nuclear Society Transactions. Winter Meeting, Vol. 87, 2002, pp. 574-579.

ICRP Publication 60 (1991) 1990 Recommendations of the International Commission on Radiological Protection, Ann. ICRP 21 (1-3) (Deutsche Ausgabe: Empfehlungen der Internationalen Strahlenschutzkommission 1990. ICRP Veröffentlichung 60. Gustav Fischer Verlag, Stuttgart, 1993).

ICRP Publication 103 (2007) The 2007 Recommendations of the International Commission on Radiological Protection, Ann. ICRP 37(2-4).

ICRP Publication 109 (2009) Application of the Commission's recommendations for the protection of people in emergency exposure situations, Ann. ICRP 39 (1).

ICRP Publication 111 (2009) Application of the Commission's Recommendations to the Protection of People Living in Long-term Contaminated Areas after a Nuclear Accident or a Radiation Emergency, Ann. ICRP 39 (3).

Raskob W., Hugon M. (Eds.) (2010) Enhancing nuclear and radiological emergency management and rehabilitation: Key Results of the EURANOS European Project, Radioprotection 45 (5), 2010. 